\title{
Improved detection of subgingival calculus by laser fluorescence over differential reflectometry
}

\author{
Fardad Shakibaie $^{1}$ (D) $\cdot$ Kristina Law $^{2} \cdot$ Laurence J. Walsh $^{1}$
}

Received: 7 November 2016 / Accepted: 18 March 2019 / Published online: 2 April 2019

(C) The Author(s) 2019

\begin{abstract}
Laser fluorescence (LF) and differential reflectometry (DR) are two new optical methods which have been used to help diagnose subgingival deposits of dental calculus. This study compared the performance of LF using the KEY3 laser system versus DR using the DetecTar system under controlled laboratory conditions designed to simulate clinical conditions as much as possible. A total of 30 extracted human posterior teeth were set in an anatomical configuration in stone typodonts with impression material replicating the periodontal soft tissues. LF was more accurate than DR (76.2\% vs. 68.2\%) and gave higher reproducibility (Bangdiwala's B statistic 0.71 vs. 0.54). The better performance of LF makes it the preferred option of the two methods for detection of subgingival dental calculus.
\end{abstract}

Keywords Subgingival calculus $\cdot$ Detection $\cdot$ Differential reflectometry $\cdot$ Laser fluorescence

\section{Introduction}

Laser fluorescence (LF) [1] and differential reflectometry (DR) $[2,3]$ are recently developed methods which have been introduced into dental clinical practice for the specific purpose of improving the ability of dentists and dental hygienists to identify hidden deposits of subgingival calculus when doing periodontal treatment. The goal of using these optical methods is to make detection faster and more reliable, since if these deposits are left on the teeth, sites of refractory inflammation will persist $[4,5]$.

To detect subgingival calculus by using the differential reflection properties of the target at two different wavelengths of light, an optical tip directs two light sources (e.g. visible red light and near infrared light) onto the root surface of the tooth. The reflected light is the used for spectral analysis, applying a processing algorithm to compare the spectra to previously stored spectra. In the DetecTar device (Ultradent, Salt Lake City, UT, USA), the dual illumination sources are LEDs which emit at either $635 \mathrm{~nm}$ or $880 \mathrm{~nm}$ [6]. The system gives an

Laurence J. Walsh

1.walsh@uq.edu.au

1 School of Dentistry, The University of Queensland, UQ Oral Health Centre, 288 Herston Road, Herston, QLD 4006, Australia

2 Faculty of Odontology, University of Malmo, Malmo, Sweden audible tone when calculus is detected, thereby providing a binary result, either yes or no.

In a past study [6], we compared the performance of calculus detection using the conventional tactile method with small probes to using DR and showed that the Detectar ${ }^{\mathrm{TM}}$ DR system gave improved accuracy and reproducibility. It was therefore of interest to undertake further work to compare DR to LF. The process of detecting the subgingival calculus using laser fluorescence typically employs a 655 -nm diode laser as the excitation source. A high-pass filter removes reflected light and ambient light (from daylight and operatory lighting), such that only near infrared light (>680 nm) will pass [7]. The near infrared fluorescence emissions are then measured using a photodetector. This forms the basic design of the DIAGNOdent Classic system [8-10], the DIAGNOdent Pen system [11-13] and the KEY3 laser system (all from KaVo, Biberach, Germany). In the latter, the fluorescence system is linked to an Er:YAG laser to give real-time feedback regarding calculus detection and calculus removal, allowing an autopilot action for automated detection and removal $[14,15]$. In recent work, we have found that the KEY3 laser has the highest accuracy and reproducibility of the three currently available LF devices for detection of subgingival calculus under defined conditions which replicate the clinical setting [16]. Hence, the purpose of the present study was to compare DR to LF, using for the latter the KEY3 system. We employed the same laboratory model as in our past work [6], to simulate 
clinical conditions and remove confounding factors from the comparison. The null hypothesis was there would be no performance differences between DR and LF methods in detection of subgingival deposits of calculus.

\section{Methods}

\section{Model preparation}

A total of 30 caries-free and restoration-free extracted teeth (18 M, 12 premolars) which had been stored in water (supplemented with $0.1 \%$ thymol) were used with ethics committee approval (reference no: 2003000040). Almost half of these extracted teeth had scattered deposits of subgingival calculus (118 out of 240 sites). All teeth were cleaned with a toothbrush under tap water. As shown in Fig. 1a, the apical third of each root was mounted into one of three stone blocks (made from non-fluorescent stone). The blocks were formed using a polyvinyl siloxane mould so that they could later be inserted into a phantom head (Frasaco, Tettnang, Germany). Each cast had 10 teeth (4 premolars and $6 \mathrm{M}$ ). Some $10-15 \mathrm{~mm}$ of the coronal and middle thirds of each tooth was exposed above the stone.

As the interface of the stone and the apical root surface may fluoresce or reflect light differently to smooth stone surfaces, the most apical $2 \mathrm{~mm}$ of the root surface should not be assessed by optical methods. To prevent false positive readings, this $2 \mathrm{~mm}$ zone was scaled using an ultrasonic scaler to eliminate any traces of subgingival calculus.

After application of a non-fluorescent water-waterbased separator (Oralube artificial saliva, Orion laboratories, Balcatta, Australia), the middle and coronal thirds of the roots were covered with a non-fluorescent Monet Clearbite2 impression material (Erskine Dental, Sydney, Australia) (Fig. 1b). After this had set, a no. 15 scalpel blade was used to trim the impression material to simulate the anatomical contours of gingival tissue. This elastic set impression material was removed from casts at the end of the study to determine the "gold standard".

\section{Optical assessment}

To ensure maximum hydration of the casts, they were soaked in water between examination sessions. On the day of use, all teeth on the stone block were covered with a thin layer of artificial saliva (Oralube, Orion laboratories, Balcatta, Australia), and the models inserted into the phantom head. The manikin was located at the level of the clinician's elbow, and a conventional halogen dental operating light was used. Prior to their use, both the KEY3 and DetecTar systems were calibrated according to the manufacturer's instructions. The scoring of the roots was undertaken by an independent examiner who had not been involved with preparing the models, with data collected for scored 8 sites per tooth using both the KEY3 and DetecTar systems. The scoring was repeated once every week for 3 weeks to evaluate intra-examiner reproducibility.

\section{Gold standard}

The gold standard was established by removing the impression material and then examining the root surfaces at $\times 20$ magnification using a stereoscopic microscope (U-PMTVC, Olympus, Tokyo, Japan). This was fitted with a 3.3 megapixel digital camera (Nikon Coolpix 995, Tokyo, Japan) so the images of the magnified root surfaces could be viewed on a monitor. In this way, the presence or absence of calculus deposits was recorded for 8 surfaces per tooth root surface (the four line angles and the four intervening middle regions).

\section{Statistical analysis}

Using the gold standard, the values for sensitivity (true positive/ (true positive + false negative)) and specificity (true negative/ (true negative + false positive)) values were calculated for DR and LF. For DetecTar examinations, the readings were either
Fig. 1 Prepared model cast. a A labial view of the mounted teeth. b A palatal view of the teeth with Monet Clearbite2 impression material serving as a replacement for soft tissues
A

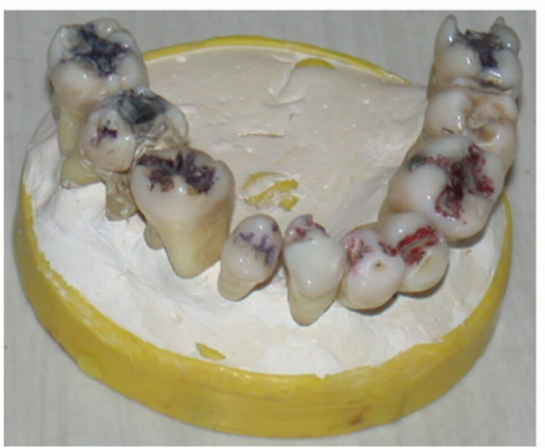

B

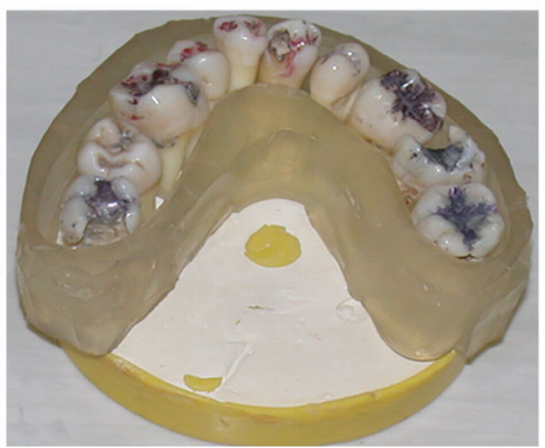


calculus present or calculus absent. For LF, the receiver operating characteristic (ROC) curves were plotted for the KEY3 laser using the true positive rates (TPR) versus false positive rates (FPR) for 10 threshold values, to determine an optimum threshold level. Using this process, a cutoff level of 7 for KEY3 was selected. This is the same as that recommended by the manufacturer. Thus, LF values less than 7 were rated as negative, and values 7 and above rated as positive, so that calculations could be made for sensitivity, specificity and accuracy. The latter was calculated as being (sensitivity + specificity) $/ 2$, and the dichotomized scores used for analysis - each one being a true or false/positives or a true or false/negatives as appropriate. In this way, the sensitivity, specificity and accuracy values were estimated. Prism 6 (GraphPad Software, La Jolla, CA, USA) was used to analyse the significance of mean differences amongst repeated measurements for two different detection systems by using upper tailed parametric paired $t$ test. Finally, Bangdiwala's B statistic was used to evaluate the intraexaminer reproducibility across the weekly repeated measurements.

\section{Results}

Data for area under the receiver operating characteristic curve for the KEY3 LF system is presented in Fig. 2. The large value for the area under the ROC curve $(80.5 \%)$ indicates excellent diagnostic performance. The ROC analysis confirmed that the most appropriate cutoff level for KEY3 was an LF reading of 7 .

As shown in Tables 1 and 3, the estimated mean values for sensitivity, specificity and accuracy respectively were greater for $\operatorname{LF}(64.7 \%, 87.7 \%, 76.2 \%)$ than for DR $(58.5 \%, 77.9 \%$,

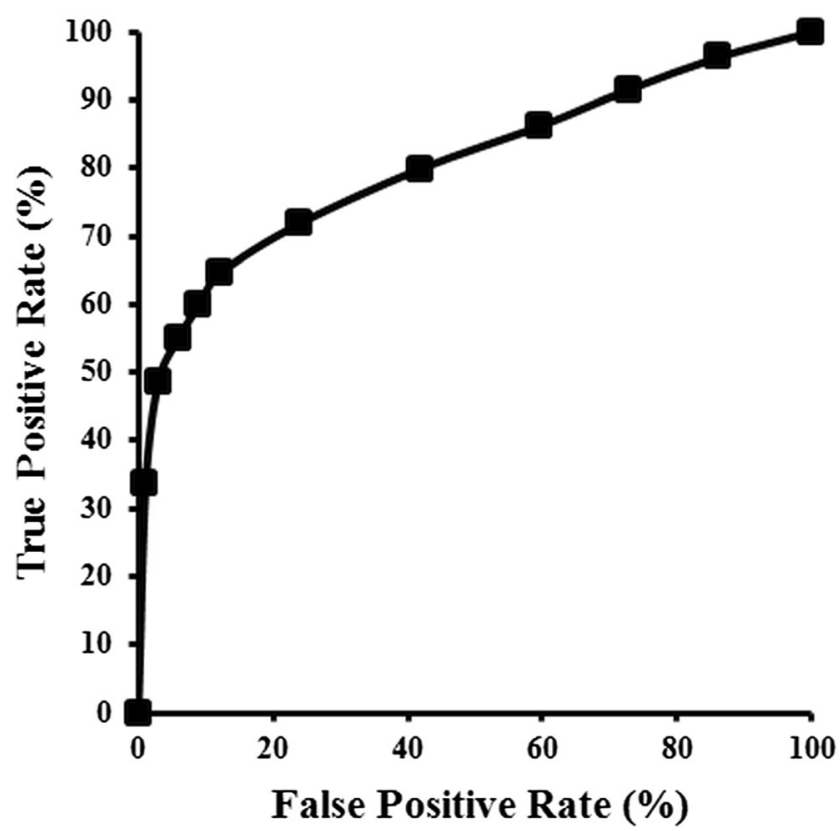

Fig. 2 Receiver operating characteristic curve for the KEY3 LF device
Table 1 Sensitivity, specificity and accuracy of optical methods

\begin{tabular}{llll}
\hline Measurements & Period & $\begin{array}{l}\text { Laser } \\
\text { fluorescence (\%) }\end{array}$ & $\begin{array}{l}\text { Differential } \\
\text { reflectometry (\%) }\end{array}$ \\
\hline Sensitivity & 1st week & 68.6 & 62.7 \\
& 2nd week & 56.8 & 50.8 \\
& 3rd week & 68.6 & 61.9 \\
Specificity & 1st week & 80.3 & 73 \\
& 2nd week & 94.3 & 84.4 \\
& 3rd week & 88.5 & 76.2 \\
Accuracy & 1st week & 74.5 & 67.9 \\
& 2nd week & 75.5 & 67.6 \\
& 3rd week & 78.6 & 69 \\
\hline
\end{tabular}

Data for laser fluorescence are based on a threshold level of 7 as set from the ROC analysis

68.2\%). These differences were significant ( $p=0.0008, p=$ 0.0104 and $p=0.0057$, respectively).

Reproducibility data are shown in Table 2 . The LF method had a higher B statistic than that of DR (0.71 with a range $0.68-0.74$, versus 0.54 with a range of $0.52-0.57$ ).

\section{Discussion}

In a past study, we had shown the value of DR over tactile probing [6] and the ability of LF to detect small deposits of calculus. Using the same laboratory assessment method, in the present study, we now show that the performance of LF exceeds that of DR. This result adds to earlier work regarding the potential usefulness of fluorescence methods $[6,17]$ and indicates that LF using the KEY3 system would be preferred for clinical use because of its high accuracy and reproducibility.

In the present study, the examiner was a 5th year undergraduate dental student with no previous experience in the application of optical methods for detecting subgingival calculus, but some clinical experience in using traditional periodontal probes. This examiner had no involvement in preparing the models used for the examination, and did not score the root surfaces at the end of the study to generate the "gold standard" data set. The question thus arises as to how much the

Table 2 Intra-examiner reproducibility

\begin{tabular}{rll}
\hline Reproducibility & Laser fluorescence & Differential reflectometry \\
\hline $\mathrm{B}_{(1,2)}$ & 0.69 & 0.52 \\
$\mathrm{~B}_{(1,3)}$ & 0.68 & 0.53 \\
$\mathrm{~B}_{(2,3)}$ & 0.74 & 0.57 \\
Mean B statistic & 0.71 & 0.54 \\
\hline
\end{tabular}

Data are based on the level of agreement between scores obtained at weeks 1, 2 and 3. The subscripts refer to the weeks being compared; thus, $\mathrm{B}_{(1,2)}$ is for data compared between weeks 1 and 2 
Table 3 Effect of experience on performance $[6,16]$

\begin{tabular}{|c|c|c|c|}
\hline Detection method & Parameter (means) & $\begin{array}{l}\text { Current } \\
\text { study }(\%)\end{array}$ & $\begin{array}{l}\text { Experienced } \\
\text { examiner }\end{array}$ \\
\hline \multirow{4}{*}{$\begin{array}{l}\text { Differential } \\
\text { reflectometry }\end{array}$} & Sensitivity & $58.5 \%$ & $75.1 \%$ \\
\hline & Specificity & $77.9 \%$ & $83 \%$ \\
\hline & Accuracy & $68.2 \%$ & $79 \%$ \\
\hline & $\begin{array}{l}\text { Reproducibility (B } \\
\text { statistic) }\end{array}$ & 0.54 & 0.61 \\
\hline \multirow{4}{*}{$\begin{array}{l}\text { Laser } \\
\quad \text { fluorescence }\end{array}$} & Sensitivity & $64.7 \%$ & $76.8 \%$ \\
\hline & Specificity & $87.7 \%$ & $95.9 \%$ \\
\hline & Accuracy & $76.2 \%$ & $86.4 \%$ \\
\hline & $\begin{array}{l}\text { Reproducibility (B } \\
\text { statistic) }\end{array}$ & 0.71 & 0.78 \\
\hline
\end{tabular}

Data for experienced examiners are taken from refs. 6 and 16

performance of the examiner could have improved with greater experience. Insight into this comes from comparisons which can be made to results using the same laboratory model but with an experienced operator (Table 3). In such hands, improvements in sensitivity, specificity, accuracy and reproducibility are evident for both DR and LF, but LF is still better than DR. This indicates that the performance advantages of LF over DR remain true regardless of operator experience.

In the present study, the clinical simulation approach used ensured that there were no confounding factors present which could have altered performance, such as the presence of dental plaque or dental caries which give strong signals with LF systems, because of their high content of fluorophores such as porphyrins [18-21]. In the past studies, we have shown that there is a strong correlation with the volume of calculus deposits [1]. How much dental plaque [22-24] and other substances [25] that may be present under clinical conditions adversely influence the performance of LF and other optical systems must be assessed through controlled clinical trials.

In the present study, a ROC analysis was used to set the diagnostic threshold for the LF system, in order to gain maximal performance. The calculated value of 7 aligns with that stated by the manufacturer [16] and has been used to determine endpoints for debridement of roots under clinical situations.

A final point of note is the potential problem posed to any optical method by fluids present in the area being assessed. These may include fluids of host origin such as blood, gingival crevicular fluid and saliva [26, 27], as well as fluids of external origin irrigated into periodontal pockets, such as hydrogen peroxide. Fluids such as hydrogen peroxide may quench fluorescence and thereby give false negatives for LF $[18,28,29]$. Under the laboratory conditions used in the present study, only a water-based non-fluorescing saliva substitute was present, and other materials that could give positive LF readings were not present or had been removed [30,31]. Once again, this highlights the need to undertake further assessments in a clinical situation.

\section{Conclusion}

This laboratory study demonstrates that laser fluorescence outperforms differential reflectometry for detecting the presence of subgingival deposits of calculus on the root surfaces of teeth.

Role of funding source This study was supported by grants from the Australian Periodontology Research Foundation and the Australian Dental Research Foundation.

\section{Compliance with ethical standards}

Conflict of interest The authors declare that they have no conflicts of interest.

Ethical approval Collection of extracted teeth for this laboratory study was carried out in accordance with the institutional ethics committee approval (reference no: 2003000040), with appropriate informed consent.

Open Access This article is distributed under the terms of the Creative Commons Attribution 4.0 International License (http:// creativecommons.org/licenses/by/4.0/), which permits unrestricted use, distribution, and reproduction in any medium, provided you give appropriate credit to the original author(s) and the source, provide a link to the Creative Commons license, and indicate if changes were made.

\section{References}

1. Shakibaie F, Walsh LJ (2014) Surface area and volume determination of subgingival calculus using laser fluorescence. Lasers Med Sci 29:519-524

2. Kasaj A, Moschos I, Röhrig B, Willershausen B (2008) The effectiveness of a novel optical probe in subgingival calculus detection. Int J Dent Hyg 6:143-147

3. Krause F, Braun A, Jepsen S, Frentzen M (2005) Detection of subgingival calculus with a novel LED-based optical probe. J Periodontol 76:1202-1206

4. Shakibaie F, Gemmell E, Bird PS (2001) A mouse model to study pathogenicity of bacteroides forsythus. Periodontology 22:5-8

5. Bird PS, Shakibaie F, Gemmell E, Polak B, Seymour GJ (2001) Immune response to Bacteroides forsythus in a murine model. Oral Microbiol Immunol 16:311-315

6. Shakibaie F, Walsh LJ (2012) Differential reflectometry versus tactile sense detection of subgingival calculus in dentistry. J Biomed Opt 17:106017

7. Shakibaie F, George R, Walsh LJ (2011) Applications of laser induced fluorescence in dentistry. Intern J Dent Clin 3:38-44

8. Hibst R, Gall R (1998) Development of a diode laser-based fluorescence caries detector. Caries Res 32:294

9. Hibst R, Paulus R, Lussi A (2001) Detection of occlusal caries by laser fluorescence: basic and clinical investigations. Med Laser Appl 16:205-213

10. Shakibaie F, Walsh LJ (2016) Laser fluorescence detection of subgingival calculus using the DIAGNOdent classic versus periodontal probing. Lasers Med Sci 31:1621-1626

11. Zhu HH, Chen QG, Lin B, Chen H (2012) Study of validity of autofluorescence-based incipient occlusal caries detection in vitro compared to DIAGNOdent pen. Laser Phys 22:614-619

12. Teo TK, Ashley PF, Louca C (2014) An in vivo and in vitro investigation of the use of ICDAS, DIAGNOdent pen and CarieScan 
PRO for the detection and assessment of occlusal caries in primary molar teeth. Clin Oral Investig 18:737-744

13. Shakibaie F, Walsh LJ (2015) DIAGNOdent pen versus tactile sense for detection of subgingival calculus: an in vitro study. Clin Exp Dent Res 1:26-31

14. Schwarz F, Sculean A, Georg T, Reich E (2001) Periodontal treatment with an Er: YAG laser compared to scaling and root planing. A controlled clinical study. J Periodontol 72:361-367

15. Schwarz F, Bieling K, Venghaus S, Sculean A, Jepsen S, Becker J (2006) Influence of fluorescence-controlled Er:YAG laser radiation, the Vector system and hand instruments on periodontally diseased root surfaces in vivo. J Clin Periodontol 33:200-208

16. Shakibaie F, Walsh LJ (2015) Performance differences in the detection of subgingival calculus by laser fluorescence devices. Lasers Med Sci 30:2281-2286

17. Shakibaie F, Walsh LJ (2016) Dental calculus detection using the VistaCam. Clin Exp Dent Res 2:226-229

18. Kurihara E, Koseki T, Gohara K, Nishihara T, Ansai T, Takehara T (2004) Detection of subgingival calculus and dentine caries by laser fluorescence. J Periodontal Res 39:59-65

19. Boston DW (2003) Initial in vitro evaluation of DIAGNOdent for detecting secondary carious lesions associated with resin composite restorations. Quintessence Int 34:109-116

20. Takamori K, Hokari N, Okumura Y, Watanabe S (2001) Detection of occlusal caries under sealants by use of a laser fluorescence system. J Clin Laser Med Surg 19:267-271

21. Buchalla W, Lennon ÁM, Attin T (2004) Fluorescence spectroscopy of dental calculus. J Periodontal Res 39:327-332

22. Walsh LJ, Shakibaie F (2007) Ultraviolet-induced fluorescence: shedding new light on dental biofilms and dental caries. Australas Dent Pract 18:56-60

23. Shakibaie F, Walsh LJ (2016) Violet and blue light-induced green fluorescence emissions from dental caries. Aust Dent J 61:464-468
24. Shakibaie F, Walsh LJ (2016) Violet and blue light-induced green fluorescence emissions from dental calculus: a new approach to dental diagnosis. Int Dent 11:6-13

25. Ha WN, Shakibaie F, Kahler B, Walsh LJ (2016) Deconvolution of the particle size distribution of ProRoot MTA and MTA angelus. Acta Biomat Odontol Scand 2:7-11

26. Shakibaie F, Walsh LJ (2015) Effect of oral fluids on dental caries detection by the VistaCam. Clin Exp Dent Res 1:74-79

27. Shakibaie F, Walsh LJ (2019) Fluorescence imaging of dental restorations using the VistaCam intra-oral camera. Aust J Forensic Sci 51:3-11

28. Hosoya Y, Matsuzaka K, Inoue T, Marshall GW Jr (2004) Influence of tooth-polishing pastes and sealants on DIAGNOdent values. Quintessence Int 35:605-611

29. Mendes FM, Pinheiro SL, Bengtson AL (2004) Effect of alteration in organic material of the occlusal caries on DIAGNOdent readings. Braz Oral Res 18:141-144

30. Shakibaie F, Walsh LJ (2017) KEY3 laser treatment applications in oral and maxillofacial surgery. J Head Neck Spine Surg 1:555574

31. Mendes FM, Hissadomi M, Imparato JC (2004) Effects of drying time and the presence of plaque on the in vitro performance of laser fluorescence in occlusal caries of primary teeth. Caries Res 38:104-108

Publisher's note Springer Nature remains neutral with regard to jurisdictional claims in published maps and institutional affiliations. 